\title{
Hormone Receptor Status in Multicentric Multifocal Infiltrating Ductal Carcinoma
}

\author{
Bayne Kilee and Jujjavarapu Rayudu* \\ Department of Surgery, Liberty University College of Osteopathic Medicine, USA
}

Received: May 29, 2018; Published: June 08, 2018

*Corresponding author: Jujjavarapu Rayudu, Department of Surgery, Tri-city Surgical Associates, Liberty University College of Osteopathic Medicine, 930 South Ave, Suite 4A, Colonial Heights, VA 23834, USA

\begin{abstract}
Multicentric multifocal infiltrating ductal carcinoma poses a different approach to diagnostic workup and treatment compared to unifocal breast cancers. This is significant especially when discussing lymph node involvement, biological receptor chemo-based therapy and breast conservative surgery. In this case, a patient presented with unilateral right bloody nipple discharge, diagnosed with multicentric multifocal hormone receptor positive infiltrating ductal carcinoma, and performed lymph node biopsy and simple mastectomy. The patient was then followed with radiation therapy. We will discuss the different presentations of breast cancers and receptor status in multifocal breast carcinomas and how we treated it from a surgical perspective.
\end{abstract}

Keywords: Multicentric; Multifocal; Infiltrating Ductal Carcinoma; Hormone Receptors; Breast Cancer

\section{Introduction}

\section{Background}

We report a case of multicentric multifocal infiltrating ductal carcinoma of the right lower breast. The two interesting topics in this case are the presentation of the patient and evaluating the receptor status in multifocal breast cancer.Breast carcinoma is the most common malignancy in females, and the most common subtype is ductal carcinoma in situ (DCIS) [1]. This type of cancer initiates in the mammary ducts and invades fatty tissues within the breast [1]. It may present as a lump or mass; skin or nipple changes; rash or redness of breast; or lymphadenopathy [1]. Our patient presented with spontaneous bloody nipple discharge and was found to have infiltrating ductal carcinoma. Spontaneous bloody nipple discharge has been found to bemore strongly associated with papilloma breast cancers [2]. Papilloma was the most common diagnosis in breasts with bloody SND and was more common than in breasts without [2]. Even though the patient had spontaneous bloody nipple discharge that was not papilloma but infiltrating ductal carcinoma, it has been reported that breast cancer can present with nipple discharge only; however, a palpable mass is usually identified in patients experiencing nipple discharge [3]. Based on the evidence of presenting with nipple discharge only, the patient would be further evaluated diagnostically in a similar manner or undergo a period of 'watchful waiting.' The conclusion of the type, stage and grade of breast carcinoma would be made to differentiate further evaluation and treatment.

\section{Case Presentation}

A 54-year-old female with past medical history of diabetes mellitus type II, hypertension, COPD, gout, hyperlipidemia, and Obstructive Sleep apnea presented to the outpatient office with right unilateral bloody nipple discharge. There was no complaint of palpable lump, history of previous trauma or infection. Patient's social history is significant for non-smoker, non-alcoholic drinker, and negative family history of breast. On physical examination, breasts were symmetric with no palpable breast masses or nipple discharge noted, and no generalized or axillary lymphadenopathy. Patient then underwent a diagnostic mammography that showed 3 vague densities in the right lower breast. An ultrasound guided biopsy was obtained of the 3 vague masses in right lower breast at the 9 o'clock position $6-8 \mathrm{~cm}$ from the nipple, the 8 o'clock position 4 $\mathrm{cm}$ from the nipple and the 3 o'clock position $8 \mathrm{~cm}$ from the nipple. Biopsy results from all 3 foci showed infiltrating ductal carcinoma. Pathologic grade of the foci was Grade 2. An MRI of the breasts was then performed which showed multicentric multifocal malignancy involving both lower quadrants of the right breast and no evidence of axillary or internal mammary adenopathy or evidence of malignancy in the left breast.

A simple mastectomy of the right breast was then performed. A sentinal lymph node biopsy was taken during the surgery and resulted negative formetastasis with negative margins. Three lymph nodes were identified as radioactive positive, sent for pathology 
and resulted negative for involvement. No further lymph node dissection was indicated at that time. Upon further pathologic evaluation of the right breast specimen, one intramammary lymph node in the axillary tail was identified as positive for micrometastasis. IHC was performed and showed ER + at 85\%, PR + at 65\% and HER2 at $0 \%$. "PROSIGNA" an oncotype test was performed with scores of $55 \%$ and $24 \%$ chance recurrence in 10 years. No further axillary dissection as indicated as the multicentric size of each tumor was small and the size of the micrometastasis was small with no extra nodal extension. Patient is undergoing radiation therapy to cover the micrometastasis. Chemotherapy was not performed given the patients co-morbidities. The post-operative course was uncomplicated. The patient was then followed by radiation oncology for radiation therapy to the right breast.

\section{Conclusion}

Breast cancer is the second leading cause of death in the USA. Widespread use of mammography has caused an increase in early detection of breast carcinoma and a decrease in mortality rates [3]. If breast cancer is suspected from a mammogram, other diagnostic tests are used to confirm it [3]. In this case, the patient presented with clinical symptoms of nipple discharge which warranted further evaluation with mammography, US guided biopsy and MRI of the breasts. Nipple discharge is a common complaint accounting for up to $5 \%$ of referrals to a breast clinic [3]. There have been some studies that show nipple discharge alone is not usually a sign of breast cancer and recommend a period of observation prior to the patient undergoing oncologic surgery [4]. While this case had nipple discharge with no associated underlying palpable breast mass, that patient did have an abnormal mammography, which conferred further workup for breast carcinoma. Cytology of the nipple discharge in this patient was not performed as there was not enough nipple discharge to collect at the time of examination.

However, per patientreport, the discharge was bloody in nature. Histopathology from previous studies revealed all patients with RBC-positive nipple discharge had benign disease, $47 \%$ had intraductal papilloma and 53\% had duct ectasia [4]. No patient with occult malignancy had RBC-positive discharge [4]. Therefore, in this patient's case, presenting with nipple discharge that was diagnosed to be infiltrating ductal carcinoma provides and interesting and unique presentation of breast carcinoma.The patients last screening mammography was unknown. It is unknown that if the patient had screening mammography's annually, a breast carcinoma would have been detected prior to onset of clinical symptoms of nipple discharge.Multicentric multifocal infiltrating ductal carcinoma presents an interesting case in concerns tomanagement. Today, breast conservation therapy is more popular in the management of women withbreast cancer.

However, with regards to multifocal breast carcinoma, it is thought that simple ormodified radical mastectomy would be a safer option to ensure complete excision of the carcinomasand any possible positive lymph nodes. Multifocal multicentric breast cancer is regularly considered arelative contraindication for breast-conserving therapy [5]. In a previous study, on whole breastspecimen showed invasive foci at more than $2 \mathrm{~cm}$ distance from the invasive primary cancer in morethan $40 \%$ of specimen [5]. Although multiple tumor foci may occur in up to $60 \%$ of mastectomyspecimens, equivalent survival outcomes were observed in prospective trials comparing BCT andmastectomy for clinically unifocal lesions, suggesting that the majority of these foci are not, or do notbecome, biologically relevant or clinically significant with appropriate treatment [5]. However,performing a lumpectomy on each mass would be disfiguring to the patient's breast and open thepossibility of incomplete extraction of all cancerous cells or the patient could possibly have othercancerous foci that are not detected on prior imaging studies.

Based on a study that compared breast-conserving therapy vs. mastectomy in multicentric multifocal breast cancer, it was concluded that breast conserving therapy was a reasonable option in selected multicentric multifocal cases, particularly those women aged 50-69 years old with small $(<1 \mathrm{~cm})$ MF tumors and without an extensive DCIS component [6]. While this patient is 54 years old, she had extensive infiltrating ductal carcinoma and would further not be a good candidate for breast conserving therapy, thus a simple mastectomy was performed.The lymph nodes were not removed in this patient as a sentinal lymph node biopsy was taken during the surgery and resulted negative for metastasis with negative margins. Three other lymph nodes that were removed in the mastectomy tissue were histopathologically evaluated and negative for metastasis.

Multifocal and mutlicentric breast cancers has been associated with an increased risk of lymph node metastases as breast tumors with multiple foci are known to have different biology [7]. One posing question in this case was if each breast tumor foci was biologically the same or different. It was found all were ER and PR positive, biologically similar, and thus may be related to the fact the patient had no lymph node involvement.Due to proper investigation and timely intervention, that patient was diagnosed and had successful removal of all foci of breast cancer. The patient is currently in good health and is undergoing radiation therapy to the right breast and axilla. Due to the patient's history of COPD, on temporary oxygen, that patient is not a good candidate for chemotherapy.

\section{References}

1. Solin L, Orel S, Hwang Wei Ting H, Harris E, Schnall M (2008) Relationship of Breast Magnetic Resonance imaging to Outcome After Breast-Conservation Treatment with Radiation for Women with EarlyStage Invasive Breast Carcnioma or Ductal Carcinoma In Situ. Journal of Clinical Oncology 26(3): 386-391.

2. Sauter ER, Schlatter L, Lininger J, Hewett JE (2004) The association of bloody nipple discharge with breast pathology. Surgery136(4): 780-785.

3. Jha K, Gupta S (2016) An atypical presentation of infiltrating ductal carcinoma. Journal of Family Medicine and Primary Care 5(4): 868-870.

4. Richards T, Hunt A, Courtney S, Umeh H (2007) Nipple Discharge: A sign of Breast Cancer? Ann R Coll Surg Engl 89(2): 124-126.

5. Nijenhuis MV, Rutgers EJ (2015) Conservative Surgery for multifocal/ multicentric breast cancer. The Breast 24(2): S96-S99.

6. Yerushalmi R, Tyldesley S, Woods R, Kennecke HF, Speers C, et al. (2012) Is breast-conserving therapy a safe option for patients with tumor multicentricity and multifocality? Annals of Oncology 23(4): 876-881.

7. Cabioglu N, Ozmen V, Kaya H, Tuzlali S, Igci A, et al. (2009) Increased lymph node positivity in multifocal and multicentric breast cancer. Journal of American College of Surgery 208(1): 67-74. 


\section{(C) (i) This work is licensed under Creative}

Submission Link: https://biomedres.us/submit-manuscript.php

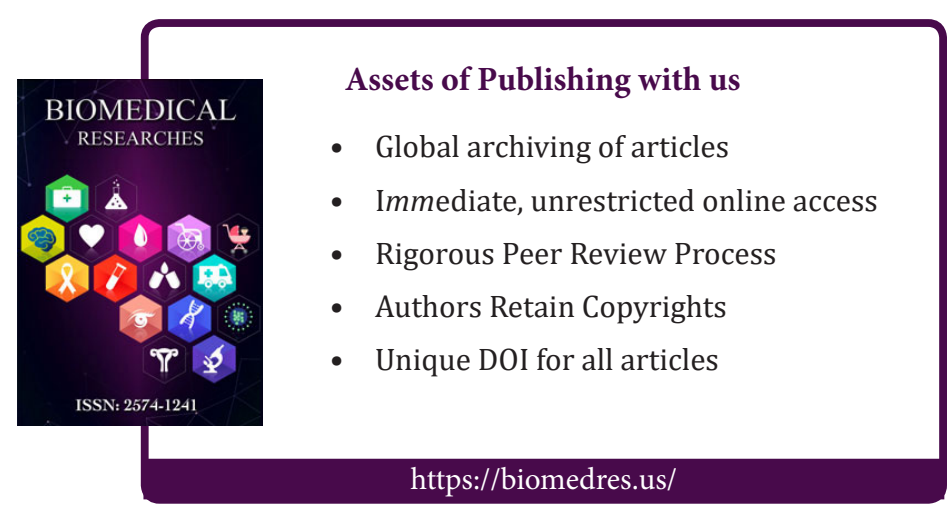

